\title{
Nonadiabatic Tapered Optical Fiber for Biosensor Applications
}

\author{
Hamid LATIFI $^{1 *}$, Mohammad I. ZIBAII ${ }^{1,3}$, Seyed M. HOSSEINI ${ }^{2}$, and Pedro JORGE ${ }^{3}$ \\ ${ }^{1}$ Laser \& Plasma Research Institute, Shahid Beheshti University, Evin, Tehran, Iran \\ ${ }^{2}$ Department of Microbiology, Faculty of Biological Sciences, Shahid Beheshti University, Evin, Tehran, Iran \\ ${ }^{3}$ INESC Porto, Rua do Campo Alegre 687, 4169-007 Porto, Portugal \\ "Corresponding author: Hamid LATIFIＥ-mail: latifi@sbu.ac.ir
}

\begin{abstract}
A brief review on biconical tapered fiber sensors for biosensing applications is presented. A variety of configurations and formats of this sensor have been devised for label free biosensing based on measuring small refractive index changes. The biconical nonadiabatic tapered optical fiber offers a number of favorable properties for optical sensing, which have been exploited in several biosensing applications, including cell, protein, and DNA sensors. The types of these sensors present a low-cost fiber biosensor featuring a miniature sensing probe, label-free direct detection, and high sensitivity.
\end{abstract}

Keywords: Biconical tapered optical fiber, nonadiabatic, refractive index, biosensor

Citation: Hamid LATIFI, Mohammad I. ZIBAII, Seyed M. HOSSEINI, and Pedro JORGE, "Nonadiabatic Tapered Optical Fiber for Biosensor Applications," Photonic Sensors, DOI: 10.1007/s13320-012-0086-z.

\section{Introduction}

A biosensor is an analytical device that couples biological recognition element with a transducer to enable rapid, accurate and sensitive detection of target analytes. Biosensors have wide applications, including biomarker detection for medical diagnostics and pathogen and toxin detection in food and water [1]. Fiber optic biosensors (FOBs) are analytical devices in which a fiber optic device serves as a transduction element. FOBs have been widely investigated because of their potential sensitivity, fast detection, biocompatibility and adaptability to a wide variety of assay conditions $[1$, 2]. Sensing in the FOBs is based on two fundamental concepts, namely spectroscopy (fluorescence and absorption) and evanescent wave field (EWF) interaction. The second concept is based on the partial overlap of the evanescent guided electromagnetic wave with a medium whose refractive index (RI) is measured. In a standard optical fiber, the intensity of the EWF decays to almost zero at the outer surface of the cladding. Thus, light propagating in these fibers is insensitive to the surroundings. With the introduction of the low-loss biconical tapered optical fiber (BTOF), light can be guided along the tapered section for optical sensing within the visible and near infrared spectral ranges. Due to their extremely small diameters of BTOFs, the amount of the penetration depth and intensity of the EWF can be significantly enhanced [3-5]. This will make it highly sensitive to the index change in the surrounding medium. So far, a number of methods have been developed for

Received: 29 July 2012 / Revised version: 8 August 2012

(C) The Author(s) 2012. This article is published with open access at Springerlink.com 
fabricating BTOFs. Sumetsky et al. [6] fabricated BTOFs by drawing a standard optical fiber in a micro-furnace comprising a sapphire tube heated with a $\mathrm{CO}_{2}$ laser. In 2006, Shi et al. [7] reported a new fabrication method for long and unbroken submicron-diameter optical fibers with a low optical loss of about $0.1 \mathrm{~dB} / \mathrm{cm}$ which were fabricated with a new drawing process by heating the conventional single mode fiber (SMF) using a designed electric strip heater. In 2010, Zhang et al. [8] presented an etch method based on surface tension driven flows of hydrofluoric acid micro-droplets for the fabrication of low-loss, subwavelength-diameter BTOFs. Tapers with losses less than $0.1 \mathrm{~dB} / \mathrm{mm}$ were demonstrated, corresponding to an order of magnitude increase in the optical transmission over previous acid-etch techniques. The etch method produced adiabatic taper transitions with minimal surface corrugations. In the same year, Pricking et al. [9] reported a model to accurately simulate the fabrication process of complex-shaped tapered fibers and fabricated tapered fibers with a sinusoidal modulated waist.

Various BTOF-based photonic devices have been reported. In 2006, Minkovich et al. [10] reported a modal interferometer built with the tapered large mode area microstructured optical fiber (MOF). In 2009, Ju et al. [11] reported the fabrication of nonadiabatic tapers in air-core photonic band gap fibers. In-fiber Mach-Zehnder interferometers were formed by utilizing such tapers and experimentally demonstrated for strain and temperature measurement. The tapers allowed the coupling between the core and surface modes to occur over a broad wavelength range. Depending on the fabricating conditions, one can fabricate BTOFs with different shapes and properties. Fiber tapers may be divided into two distinct categories: adiabatic and non-adiabatic [12-14]. A tapered fiber can be considered as adiabatic if the main portion of the power remains in the fundamental mode and does not couple to higher order modes as it propagates along the taper. For this structure, the relative local change in the taper radius has to be very small (small taper angle), as shown in Fig. 1(a). It has been shown that the nonadiabatic tapered optical fiber (NATOF) as shown in Fig. 1(b) can be made so that coupling occurs primarily between the fundamental mode of the unpulled fiber and the first two modes of the taper waveguide, where, due to the large difference of the RIs of air and glass, the taper normally supports more than one mode. The result of back and forth coupling between the single mode of the fiber and the two (or more) modes of the taper is oscillation in the spectral response of the taper. Therefore, the NATOF behaves as a modal interferometer.

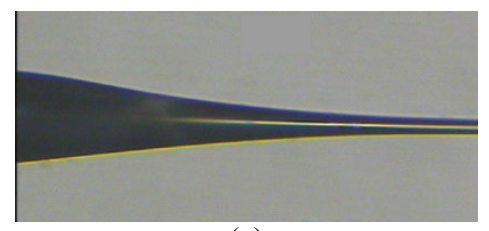

(a)

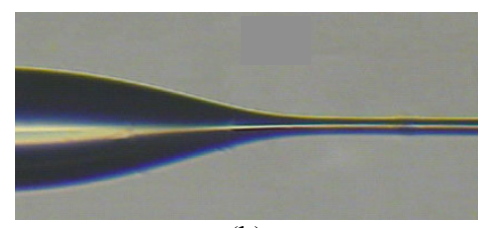

(b)

Fig. 1 Photos of different structures of the BTOF: (a) adiabatic tapered and (b) non-adiabatic tapered [12].

Adiabatic tapered fibers have been used for coupling light in and out of other sensing structures for bi-chemical sensing such as liquid-core optical ring-resonators [15], micro-disks [16], and microspheres [17-19]. Also, the adiabatic tapered fiber has been used for the fabrication of the high sensitive surface plasmon resonance (SPR) sensor to the RI.

Typical structures of BTOF sensors are shown in Fig. 2. Figure 2(a) depicts the simplest straight BTOF sensor, in which the thickness of the tapered section is usually close to or less than the operating wavelength. Since the high fractional guiding mode of the BTOF propagates outside the fiber, the transmission intensity of a BTOF depends on the 
properties of the ambient medium, thus these fibers have been applied to the RI, humidity, and chemical/ biological sensing. Figure 2(b) shows a U-shaped tapered optical fiber, and Fig. 2(c) shows optical sensors based on BTOF loop/knot/coil resonators that are assembled by micromanipulation.
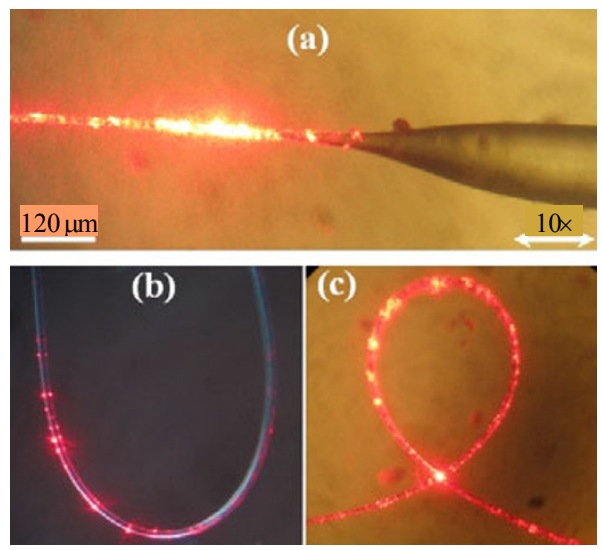

Fig. 2 Typical structures of BTOF sensors: (a) straight BTOF, (b) U-shaped sensor based on the BTOF, and (c) optical sensors based on BTOF loop/knot resonators.

Some of the NATOF sensors reported can measure the strain [20], temperature [12, 20, 21], humidity [22] and RI [12, 20]. As many unlabeled optical biosensors are essentially RI sensors, the NATOF sensor can be utilized as an unlabeled biosensor for numerous applications in health care, environmental protection, food safety, and petrochemical industries. From a detection standpoint, some advantages of the NATOF sensor include the exposure of the EWF beyond the surface of the sensing region, fast and real-time response.

In the past few years, NATOF biosensors have been investigated in our laboratory as an alternative detection device for biomolecules. This review intends to describe the recent advances using the NATOF sensor in the biochemical application. Special focus will be given to the RI measurement and label free biosensor.

\section{Modeling of the evanescent wave sensor based on the BTOF}

A large penetration depth of an evanescent wave is the key to success for developing an ultra high-resolution, fiber-based evanescent wave biosensor. Several investigators have attempted to increase the penetration depth of the EWF and facilitate mode coupling.

In 1981, Kawasaki et al. [23] performed the tapering of SMFs in the fabrication of most kinds of fused fiber couplers.

In 1986, Burns et al. [24] showed that the ultimate loss mechanism in a fiber taper was due to the cutoff of the fundamental mode from the core. The lost power became a cladding mode guided by the air-cladding interface and could not be recaptured by the core. In 1990, Bobb et al. [25] reported the fabrication of biconically tapered SMFs for bending measurement. In 1991, field evolution along longitudinally nonuniform finite-cladding fibers of the circularly symmetric cross section was analyzed in terms of coupled modes by Gonthier [26].

In 1999, Moar et al. [27] reported fabricating tapered fibers using a flame elongation technique. The EWF distribution surrounding the taper region was measured directly using a scanning near-field optical microscope, and results were found to be in good agreement with predictions made using a finite difference beam propagation method.

In 2002, Fielding et al. [28] investigated numerically and experimentally how a point source of radiation coupled light to the $\mathrm{HE}_{11}$ mode of a nonadiabatic and tapered single-mode fiber for the biosensor application.

In 2003, a theoretical analysis of the coupling mechanism that took place inside a taper was studied by Matias et al., and a novel expression for the coupling coefficient was derived. The studies done by Bobb et al. showed an expression for the same coefficient, but only under static conditions. The expression derived by Matias et al. was a generic expression and was valid under static or dynamic conditions. It was demonstrated that the optical power coupled at the output of a taper depended on the time-dependent bending angle of 
the taper, hence showing its usefulness for vibration, displacement sensors or modulators [29]. In 2005, Ahmad et al. [30] using a ray-tracing model showed theoretically that a large penetration depth of an evanescent wave was the key to success for developing an ultra high-resolution fiber-based evanescent wave biosensor. Tapering the fiber and launching light at an angle had the potential for increasing the penetration depth of evanescent wave manifolds. Evanescent wave penetration depths of the order of the size of living cells have been achieved by optimizing the parameters relating to the geometry of tapered fibers.

\section{RI sensor}

RI measurement in small volumes plays a vital role in many areas of biophysics, biochemistry and biomedicine. For example, the RI can be used to determine the concentration of sugar or proteins. A number of BTOF-based RI sensors were reported recently. The sensors can be classified according to the following categories: surface plasmon resonance sensors based on adiabatic BTOF and NATOF RI sensors.

\subsection{Surface plasmon resonance sensors based on the adiabatic BTOF}

In 1995, a novel SMF surface plasma wave sensor was employed which operated over an extremely broad spectral range reported by Tubb et al. [31]. This was achieved by coating a gradually tapering SMF. The gradual change in the taper cross-section resulted in distributed phase matching between the fiber mode and the surface plasma wave, which permitted a plasma wave to be excited over a spectral range from $600 \mathrm{~nm}$ to $1000 \mathrm{~nm}$. The RI resolution of the sensor was about $5 \times 10^{\square 4}$. In 2006, Esteban et al. [32] presented a configuration for SPR sensors based on uniform-waist tapered optical fibers and reflective elements. The fiber was tapered fulfilling the adiabatic criterion, coated with a multilayer asymmetrically on the uniform waist of the fiber, and a mirror was produced at the fiber tip. In this way, the sensor operated in a reflective mode, more convenient for dip probes. When these sensors were spectrally interrogated, a high sensitivity of $10^{-4} \mathrm{RIU} / \mathrm{nm}$ was attained. In 2008, Verma et al. [33] reported a comprehensive theoretical model to evaluate the influence of the tapering ratio on the performance of a fiber optic SPR sensor, where a high taper ratio was recommended with an exponential-taper profile to design a highly sensitive fiber optic SPR probe. In 2010, Díaz-Herrera et al. [34] reported that multiple surface plasma waves could be excited in optical fibers at the $1.5-\mu \mathrm{m}$ optical communication region for the range of RIs of aqueous media using doubly deposited tapered fiber structures. Figure 3 shows the experimental setup. A sensitivity about $5000 \mathrm{~nm} / \mathrm{RIU}$ at $1500 \mathrm{~nm}$ was obtained, which was higher than the same setup operating in the $800-\mathrm{nm}$ region, whose sensitivity was about $3000 \mathrm{~nm} / \mathrm{RIU}$.

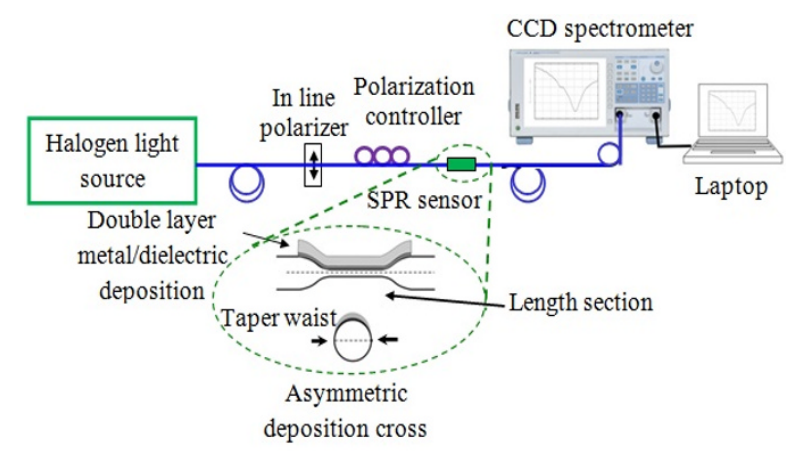

Fig. 3 Experimental setup for the characterization of the SPR sensors proposed by Díaz-Herrera et al.[34].

Later on that year, the same group reported using an adiabatic taper with a chemical treatment of the fiber for the SPR application, and an overall sensitivity of around $2400 \mathrm{~nm} / \mathrm{RIU}$ was obtained [35].

\subsection{RI sensor based BTOF}

In 1984, Kumar et al. [36] proposed a novel fiber optic refractometer using a tapered multimode fiber (MMF). It could measure RIs to a high degree of accuracy and required a very small amount of the 
sample to perform the measurement. It was shown that, in principle, this refractometer could be designed to measure any RI less than that of the core with a better sensitivity than that of the earlier reported fiber refractometers. In 2004, Villiatoro et al. [37] presented a new fiber optic RI sensor. It was based on the radiation losses introduced by the sample medium in the tapered region of an MMF. A simple light transmission setup was used to test some samples. It was demonstrated that the sensor was suitable for measuring RIs in the range of 1.36 to 1.46 , as well as RI changes below $10^{-4}$. In 2011, Wang et al. [38] proposed and experimentally demonstrated an enhanced EWF fiber refractometer based on a tapered MMF spliced between two SMFs. For RI measurements within the range of 1.33 to 1.44 , a maximum sensitivity of $1913 \mathrm{~nm} / \mathrm{RIU}$ was achieved with a $30-\mu \mathrm{m}-\mathrm{MMF}$ taper waist diameter. Polynkin et al. [39] reported a simple optical sensing device capable of measuring the RI of liquids propagating in microfluidic channels. The sensor was based on a submicrometer tapered fiber immersed in polydimethylsiloxane (PDMS). The sensor could operate both as a highly responsive on-off device and in the continuous measurement mode, with an estimated accuracy of $5 \times 10^{-4}$ for measurement. In 2006, Kieu et al. [20] presented an SMF BTOF sensor for measurement of the displacement, temperature, and RI. A sensitivity of $1.42 \times 10^{-5}$ was achieved using tapers made from a standard single-mode fiber. In 2008, the behavior of tapered graded-index polymer optical fibers was analyzed computationally based on ray tracing and experimentally for different RIs of the surrounding medium by Arrue et al. [40]. In the same year, Leung et al. [41] reported the tapered fiber sensors at $1310 \mathrm{~nm}$ and $1550 \mathrm{~nm}$ were sensitive to glucose with the concentrations of $0.01 \mathrm{~g} / \mathrm{ml}$ to $0.1 \mathrm{~g} / \mathrm{ml}$ at $0.5 \mathrm{ml} / \mathrm{min}$, which gave a sensitivity in terms of dB/RIU. In 2010, Cohoon et al. [42] reported the doubling of sensitivity for these sensors with two passes through the tapered region, which became possible through the use of sensitive and high-dynamic-range photodetectors.

Figure 4 presents the experimental setup. The sensitivity was improved from $0.43 \mathrm{~dB} / \mathrm{vol} \%$ in a single-pass case to $0.78 \mathrm{~dB} / \mathrm{vol} \%$ with two passes through the taper. The RI detection limit was estimated to be $1.2 \times 10^{-5} \mathrm{RIU}$ and $0.6 \times 10^{-5} \mathrm{RIU}$ in the single and double-pass schemes, respectively.

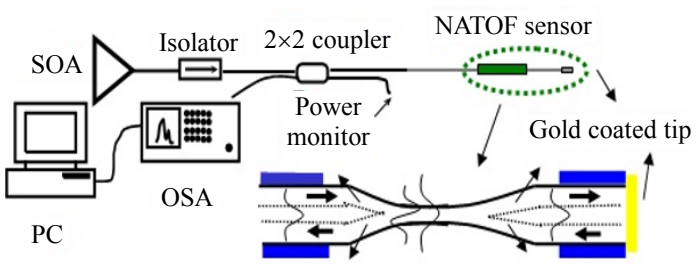

Fig. 4 Experimental setup for doubling the sensitivity of the NATOF sensors with two passes through the tapered region, as reported by Cohoon et al. [42].

In 2011, Rahman et al. [43] reported a simple tapered plastic multimode (PMM) fiber optic sensor for continuous monitoring of salinity based on different concentrations of sodium chloride in de-ionized water. The tapered PMM fiber was fabricated using the heat-pulling method to achieve a waist diameter and a length of $0.187 \mathrm{~mm}$ and $5 \mathrm{~mm}$, respectively. When the solution concentration changed from $0 \%$ to $12 \%$, the output voltage of the sensor increased linearly from $0.109 \mathrm{mV}$ to $1.142 \mathrm{mV}$, with a sensitivity of $0.0024 \mathrm{mV} / \%$ and a linearity of more than 98\%. Beres et al. [44] reported sensitivities for the tapered plastic optical fiber (POF) in the RI range of 1.330 to 1.393 . Sensitivities for straight tapers and U-shaped tapers were approximately $6.1 \mathrm{mV} / 10^{-3} \mathrm{RIU}$ and $12.1 \mathrm{mV} / 10^{-3} \mathrm{RIU}$, respectively. The uncertainties in RI measurements were $12.2 \times 10^{-3} \mathrm{RIU}$ and $1.42 \times 10^{-3} \mathrm{RIU}$, respectively. U-shaped POF tapers are being used for detection of target cells.

In order to diversify the applications, complex tapered fiber structures have been developed. In 
2005, Ding et al. developed a refractometer based on a pair of long period gratings (LPGs) into two sides of the taper region, with a RI resolution of $\pm 5.8 \times 10^{-6}$ for a measurement system with a normal spectral resolution of $1 \mathrm{pm}$ and a RI in the range of 1.3333 to 1.3624 [45]. In a follow up study, Allsop et al. fabricated a single LPG into a BTOF. A limit of the resolution of the RI, $\pm 8.5 \times 10^{-5}$, was obtained for solutions with the RI in the range of 1.330 to 1.335 [46]. Some fiber Bragg gratings-based tapered fibers were used to measure the RI changes in the ambient environment as low as $2.5 \times 10^{-5}$ [47]. As shown in Figs. 5(a) and 5(b), Liang et al. [48] reported two different FBG technologies with a chemical etch-erosion procedure and demonstrated two types of RI sensors using SMFs. The first RI sensor device was an etch-eroded single FBG with a radius of $3 \mu \mathrm{m}$. The second RI sensor device was an etch-eroded fiber Fabry-Pérot interferometer (FFPIs). Both configurations had a narrow spectral feature, however the FFPI sensor had a higher sensitivity than the FBG sensor and could detect a RI variation of $1.4 \times 10^{-5}$. Fang et al. [49] fabricated FBGs in BTOFs using femtosecond laser pulse irradiation. Such a grating could be directly exposed to the surrounding medium without etching or thinning treatment of the fiber, thus possessing high RI sensitivity while maintaining excellent reliability.

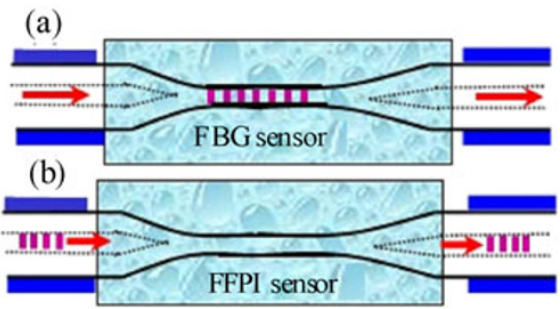

Fig. 5 Schematic view of a NATOF: (a) single fiber Bragg grating sensor and (b) FFPI sensor.

Depending on the fiber diameter, the micro fiber grating supported a number of propagation modes, and the higher-order mode offered the higher RI sensitivity. The highest sensitivity obtained was $231.4 \mathrm{~nm} / \mathrm{RIU}$ with a RI of 1.44 when the fiber diameter was about $2 \mu \mathrm{m}$. In 2010, an SMF NATOF sensor was utilized for sensing the variation in the RI with the concentration of D-glucose in de-ionized water and measuring the RI of amino acids (AAs) in carbohydrate solutions by Zibaii et al. [22]. Figure 6 presents the setup used for measuring the AAs' RI. The NATOF was fabricated by the heat pulling method, using a $\mathrm{CO}_{2}$ laser. A typical NATOF is shown in Fig. 7 with a tapered length of around $843 \mu \mathrm{m}$.

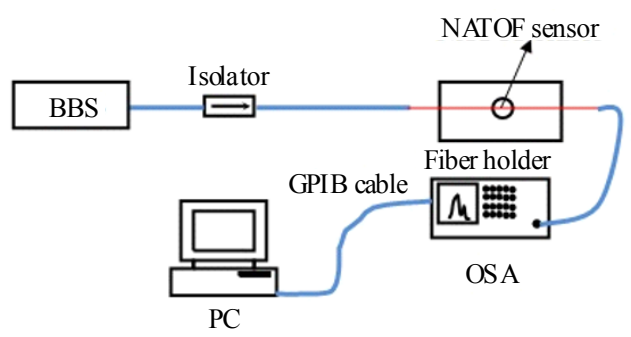

Fig. 6 Instrumentation setup for measuring the RI of $\alpha$-amino acids in the aqueous D-glucose and sucrose solution [12].

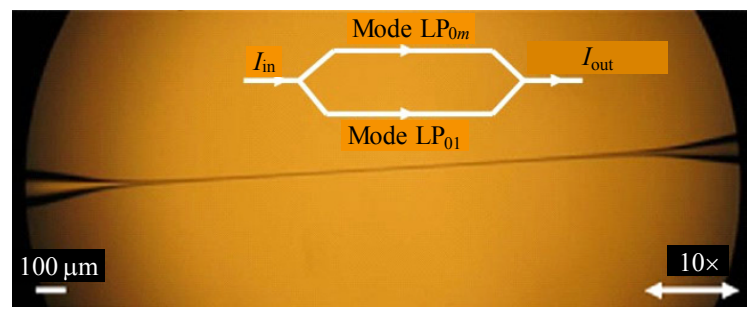

Fig. 7 A non-adiabatic tapered fiber with a taper length of $843 \mu \mathrm{m}$ and a waist diameter of $11 \mu \mathrm{m}$.

Figure 8 shows the output spectrum from the tapered fiber. The limit of detection of the NATOF sensor was $55 \mathrm{ppm}$ for a D-glucose concentration ranging from 0 to $80 \mathrm{mg} \cdot \mathrm{ml}^{-1}$, and the limit of detection of the RI measurement corresponding to these concentrations in the range from 1.3330 to 1.3447 was $8.2 \times 10^{-6}$ as a refractometer sensor. This method showed a rewarding ability in understanding the basis of biomolecular interactions in biological systems. Later on that year, the same group proposed inserting the NATOF sensor into a fiber loop mirror (FLM) for tuning its sensitivity towards the RI [50]. Figure 9 illustrates the experimental setup which consisted of an optical broadband 
source, a fiber loop mirror, a NATOF and an optical spectrum analyzer (OSA).

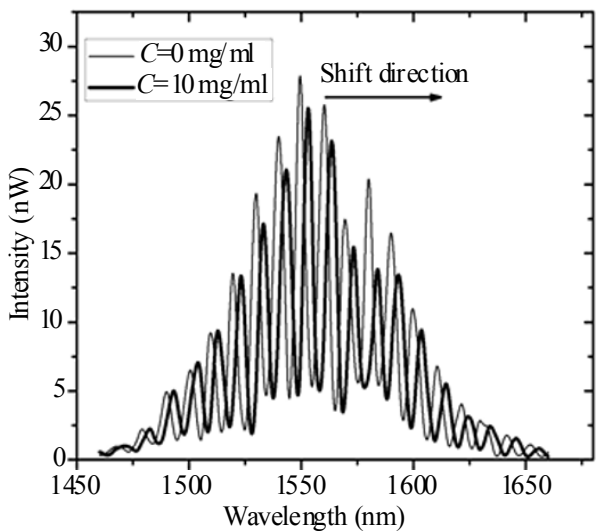

Fig. 8 Output spectrum from the NATOF for $C=0 \mathrm{mg} \cdot \mathrm{ml}^{\square 1}$ and $C=10 \mathrm{mg} \cdot \mathrm{ml}^{-1}$ for the $\mathrm{D}$-glucose solution at $23{ }^{\circ} \mathrm{C}$.

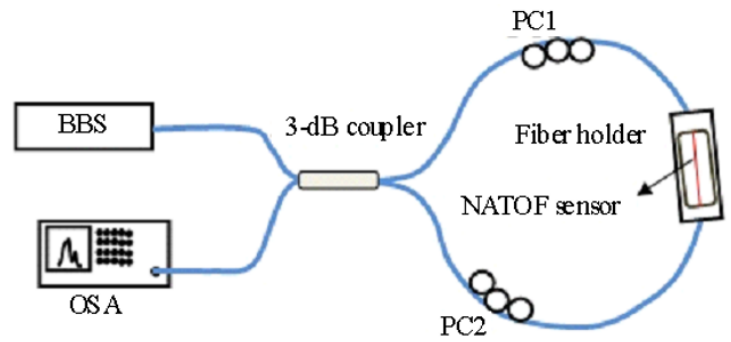

Fig. 9 Schematic of a NATOF inserted in the fiber loop mirror [50].

The taper-fiber loop mirror (TFLM) was formed by a 3-dB optical coupler with the low insertion loss, two-polarization controllers (PCs) and a NATOF which was placed between PCs. The PCs were used to optimize the visibility of the fringe pattern of the interferometers and to excite the high order cladding modes. The beam traveled towards the $3-\mathrm{dB}$ coupler at port 1 , and the incident beam was split into two propagating beams. Fifty percent of the incident optical power was directed to port 3 and traveled clockwise around the loop; the remaining fifty percent was directed to port 4 and traveled counterclockwise with a phase difference of $\pi / 2$. The two beams contrarily passed through PCs and NATOFs, and the transmission beam exited the $3-\mathrm{dB}$ coupler through port 2 .

When varying any of the PCs, various cladding modes were excited in each arm of the TFLM and resulted in different phase interference for clockwise and counterclockwise beams. Figure 10 shows the results for three settings of the PCs and considers the excitation of different cladding modes. It showed high order cladding modes had a low visibility because of the low mode power. Figure 11 shows a wavelength shift in the transmission peak 1 versus the RI in the 1.3380 to 1.3510 range for three different positions of the PCs in Figs. 10(a), 10(b), and $10(\mathrm{c})$. It is apparent that the peak linearly shifts toward longer wavelengths as the RI increases. For peak 1 , the sensing curves have good coefficients of determination of $0.99466,0.99299$ and 0.99801 with the corresponding sensitivities of $876.24 \mathrm{~nm} / \mathrm{RIU}$, $1023.96 \mathrm{~nm} / \mathrm{RIU}$, and $1233.07 \mathrm{~nm} / \mathrm{RIU}$ for the PC settings in Figs. 10(a), 10(b), and 10(c), respectively. The sensitivities for the cases in Figs. 10(b) and 10(c) are compared with the case in Fig. 10(a), and a change of more than $14 \%$ and $24 \%$, respectively, is found. It showed high order cladding modes were more sensitive to the RI. The sensitivity of the sensor was enhanced about $80 \%$ and compared to the previous work using a Hi-Bi FLM with the chemical etched D-type fiber for the RI measurement [51]. In addition, the sensitivity of the TFLM could be controlled, and it showed a great potential for several applications, particularly to monitor chemical and biological parameters in natural environments.

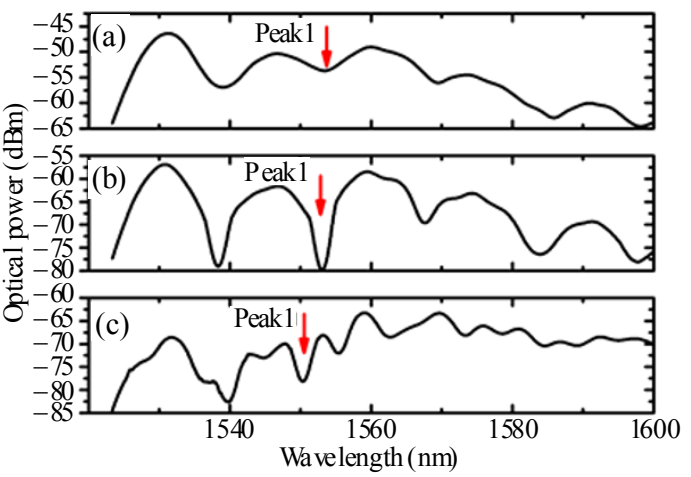

Fig. 10 Spectral response of the NATOF in the water solution at room temperature for three different positions (a), (b), and (c) of the PCs. 


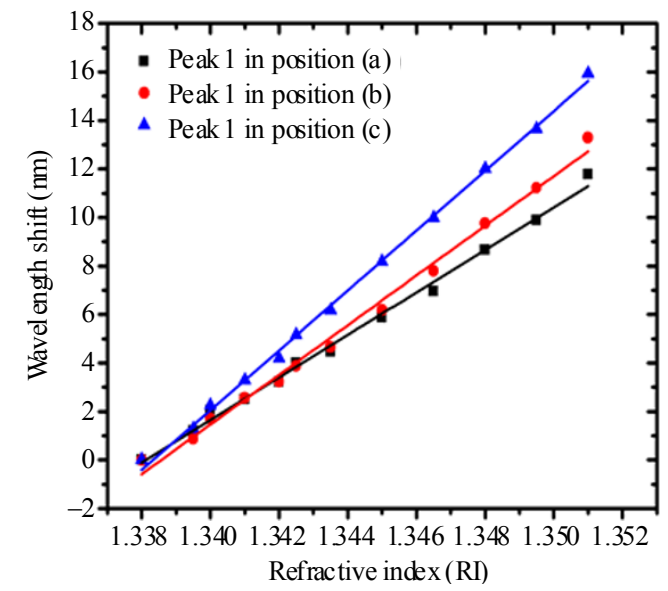

Fig. 11 Wavelength shift in transmission peak 1 versus the RI for three different positions of the PCs in Figs. 10 (a), 10(b), and $10(\mathrm{c})[50]$.

\section{Biosensor application}

Generally, there are two detection protocols that can be applied to optical biosensing: fluorescence-based detection and label-free detection. In fluorescence-based detection, either target molecules or biorecognition molecules are labeled with fluorescent tags. The intensity of the fluorescence indicates the presence of the target molecules and the interaction strength between target and biorecognition molecules. Even though fluorescence-based detection is extremely sensitive, with a detection limit down to a single molecule [52], it has some disadvantages such as the challenges presented for quantitative analysis, difficulty of finding an appropriate label that functions equivalently for all molecules [53], negative effects on the biomolecules, the time-consuming pretreatment and high operation costs.

In contrast, in label-free detection, target molecules are not labeled or altered and are detected in their natural forms. This type of detection is relatively easy and cheap to perform, and allows for quantitative and kinetic measurement of molecular interaction. Additionally, as discussed later, some label-free detection mechanisms measure RI changes induced by molecular interactions, which are related to the sample concentration or surface density. As a result, the detection signal does not scale down with the sample volume, which is attractive for the ultra small detection volume.

Despite all these differences between fluorescence-based and label-free detection, both protocols are being widely used in optical biosensors and provide vital and complementary information regarding interactions among biomolecules, which makes optical sensors more versatile than other types of sensing technologies.

\subsection{Fluorescence-based biosensors}

In 1996, James et al. [54] developed a fluorescence tapered fiber sensor based on competitive assay for detecting lipopolysaccharide (LPS) endotoxin as an immune stimulant of sepsis. In the study, LPS from Escherichia coli (E. coli) was detected at concentrations as low as $10 \mathrm{ng} / \mathrm{ml}$, in $30 \mathrm{~s}$. The fluorescent labeled LPS was introduced to the fiber and attached to the Polymyxin B as a recognition molecule. The unlabeled LPS was then introduced and competed with the labeled LPS for the binding sites on the Polymyxin B. As the unlabeled LPS concentration increases, fluorescence decreases.

In 1996, Hale et al. [55] developed a fluorescent optical fiber loop sensor to detect antibodies to IgG. A bent tapered fiber biosensor was used with a two-step sandwich assay. IgG was labeled with the fluorescent dyes fluorescein isothiocyanate or tetramethyl rhodamine. In the first step of the assay, the tapered fiber was silanized so that the unlabeled IgG was attached to the sensing region covalently. Then, the antibody to the IgG was bound to the sensing region due to the presence of the attached IgG. Finally, the labeled IgG was introduced, and the argon ion laser radiation entered the evanescent region to excite the fluorescent dye. The fluorescence emitted by the dye was coupled back into the fiber and was the direct measurement of the IgG concentration. A concentration of $75 \mathrm{pg} / \mathrm{ml}$ was detected with this method. 
In 1997, Narang et al. [56] reported a fluorescent tapered fiber-optic biosensor for detecting ricin as a toxic protein at pictograms $/ \mathrm{ml}$ levels with a sandwich immunoassay scheme. Firstly, an anti-ricin IgG was immobilized onto the surface of an optical fiber by silanization and avidin-biotin linkage. Then, ricin was introduced into the vicinity of the sensor. Finally, a Cy5-labeled secondary antibody was used to complete the sandwich immunoassay. The assay using the avidin-biotin linked antibody demonstrated the higher sensitivity and wider linear dynamic range than the assay using antibody directly conjugated to the surface. The linear dynamic range of detection for ricin in the buffer using the avidin-biotin chemistry was $100 \mathrm{pg} / \mathrm{ml}-250 \mathrm{ng} / \mathrm{ml}$. The limit of detection for ricin in the buffer solution was $100 \mathrm{pg} / \mathrm{ml}$, and in river water, it was $1 \mathrm{ng} / \mathrm{ml}$. At concentrations of ricin greater than $50 \mathrm{ng} / \mathrm{ml}$, there was strong interaction of ricin with the avidin due to the lectin activity of ricin. This interaction was significantly reduced using fibers coated with neutravidin or by adding galactose to the ricin samples.

Pilevar et al. presented an all-fiber hybridization assay sensor based on the fluorescence adiabatically tapered single-mode fiber probe for detecting Helicobacter pylori total Ribonucleic acid (RNA) [57]. Probe oligonucleotides were cross-linked to the tapered surface. Real-time hybridization of near-infrared fluorophore IRD. 41-labeled oligonucleotide at various concentrations to the surface bound probes was performed. Using 20-mers as probes, complementary oligonucleotides at lower concentration than $\mathrm{nM}$ were detected. Sandwich assays were performed with Helicobacter pylori total RNA to determine if the sensor could detect bacterial cells using rRNA as the target, and it was found that this sensor could detect $H$. pylori RNA in a sandwich assay at $25 \mathrm{pM}$.

As shown in Fig. 12, Haddock et al. [58] developed a technique using the EWF of tapered fibers for rapid, convenient, and accurate sensing of biomolecules and cells using small volumes of analytes in the range of $0 \square 150 \mu \mathrm{L}$. Using an analytical grade spectrofluorometer nicotinamide adenine dinucleotide (NADH), nicotinamide adenine dinucleotide phosphate (NADPH), and Chinese hamster ovary $(\mathrm{CHO})$ cells at various concentrations were measured. The detection limit of the fiber for CHO was $0.1 \times 10^{6}$ cells $/ \mathrm{ml}$. It was found that the limits of detection of the taper were $0.2 \mu \mathrm{M}$ of $\mathrm{NADH}$ and $0.5 \mu \mathrm{M}$ of NADPH. The limit of detection for the cuvette was $3 \mu \mathrm{M}$ for both NADH and NADPH.

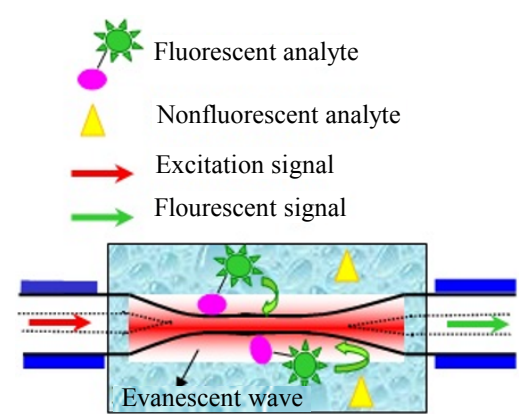

Fig. 12 Illustration of evanescent field fluorescence sensing approaches.

\subsection{Label-free biosensors}

\subsubsection{Bacterial growth rate}

In order to detect the growth of $E$. coli O157:H7, an intensity-based evanescent sensor was investigated by Ferreira et al. [59]. The sensor was fabricated by chemically etching an MMF, and the sensing was based on the interaction of the bacteria with the EWF as well as the attenuation light. The power loss was proportional to the intrinsic bulk absorption and scattering, which depended on the concentration of the bacteria. The sensitivity of this sensor was $0.016 / \mathrm{dB} / \mathrm{h} / N_{\mathrm{o}}$, where $N_{\mathrm{o}}$ ranged from 10 to 800 and was the initial number of the bacteria. In a follow up study by Rijal et al., E. coli O157:H7 was covalently bonded to the surface of a tapered fiber via an antibody, and concentrations as low as 70 cells $/ \mathrm{mL}$ was detected by changes in the intensity [60]. 
In 2006, Maraldo et al. used a tapered fiber sensor to detect the growth of E.coli JM 101 [61]. The tapered surface was coated with poly-L-lysine (PLL), and E. coli JM 101 expressing green fluorescent protein was immobilized. The growth was monitored by light transmission through the tapered fiber. The transmission decreased exponentially with the cell growth on the tapered surface.

In 2010, Zibaii et al. [62] reported real-time monitoring of the E. coli $\mathrm{K}-12$ growth in an aqueous medium by the NATOF biosensor. The bacteria were immobilized on the tapered surface using PLL. The experimental setup is shown in Fig. 13. It consisted of a distributed feedback laser (DFB) with the maximum rated output power of $20 \mathrm{~mW}$ and peak wavelength of $1558.17 \mathrm{~nm}$. The laser beam which arrived at the tapered fiber sensor after passing a dual stage isolator and a $2 \times 2$ coupler was detected by photodiode 2 (PD2), which was the sensor signal. The reflected light of the coupler was then recorded by photodiode 1 (PD1) and labeled as the reference beam. Finally, the data obtained from the two identical detectors were delivered to an analog to digital converter and was processed by the LabVIEW software.

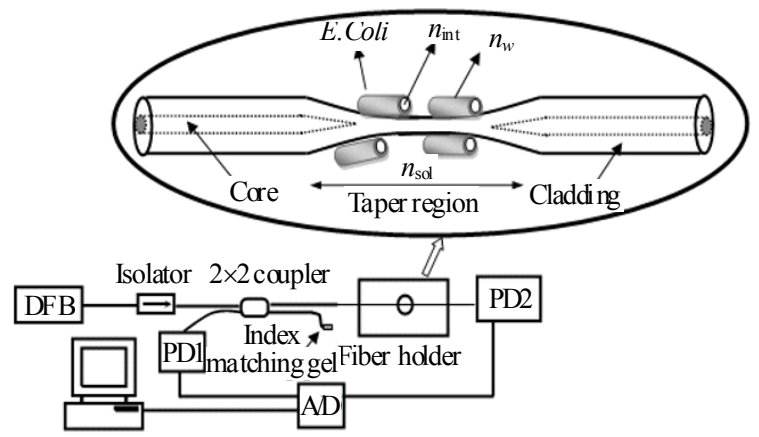

Fig. 13 Real-time monitored bacterial growth rate schematic proposed by Zibaii et al. [62].

The measured specific growth rate by the NATOF biosensor and pour plate method as shown in Fig. 14 were found to be $-0.17 \pm 0.01 \mathrm{~h}^{-1}$ and $0.33 \pm 0.02 \mathrm{~h}^{-1}$, respectively, for a 1.6 - $\mathrm{h}$ period at room temperature. When the bacteria were grown on the taper, the fiber optic biosensor exhibited strong transmission changes at $1558 \mathrm{~nm}$, which was proportional to the variation in the cell concentration. Therefore, it could be inferred that the transmission through the fiber was decreased by an increase in the average surface density of the cells on the tapered fiber. An increase in the average density of the bacteria changed the dielectric constant of the new cladding (E. coli in the glucose growth medium) and the RI of the tapered section, as well.

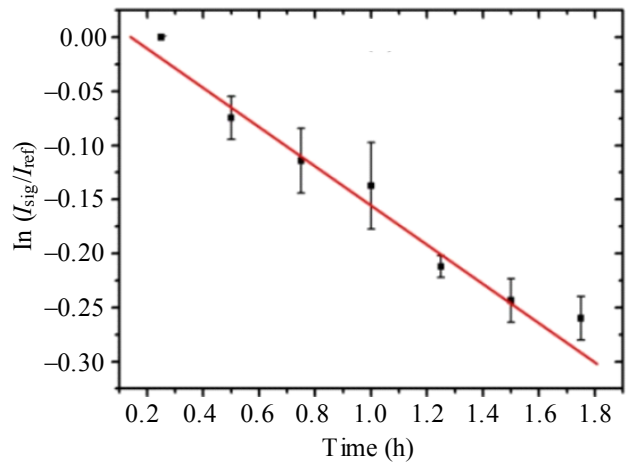

(a)

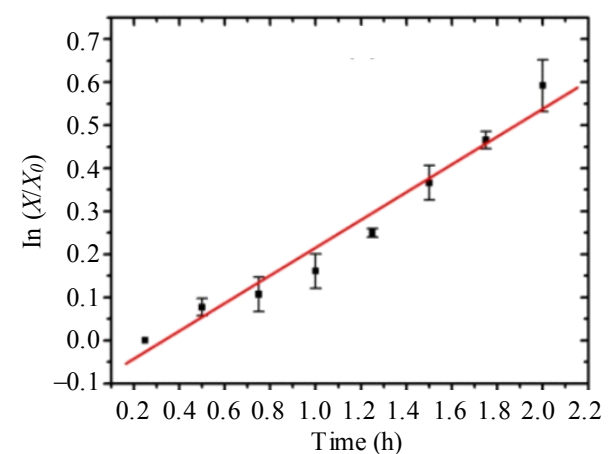

(b)

Fig. 14 Bacterial growth rate measurement: (a) plot of sensor response versus time during $E$. coli growth on the tapered fiber and (b) growth rate measurement with colony counting method [62].

The sensitivity of the current setup was measured to be $60 \mathrm{E}$. coli $\mathrm{mm}^{-2}$, which corresponded to $17 \times 10^{-12} \mathrm{~g} \cdot \mathrm{mm}^{-2}$ dry-mass loading or a total of 10 bacteria bound to the tapered surface. The new bacterial growth measurement system provided numerous advantages such as the small size, rapid real-time and label free performance intended for small analyte volumes over other methods like plate counting, optical density, and dry weight. 


\subsubsection{Porotoein detection}

In 2007, Tazawa et al. [63] demonstrated fiber couplers to detect streptavidin. In this case, two identical optical fibers were fused together and tapered to a diameter of $9 \mu \mathrm{m}$. The transmission spectrum was sinusoidal and showed a RI sensitivity close to $70 \mathrm{~nm} \cdot \mathrm{RIU}^{-1}$ with a detection limit of $4 \times 10^{-6}$ RIU. This sensor was then able to detect streptavidin with concentrations between $0.5 \mu \mathrm{g} \cdot \mathrm{ml}^{-1}$ and $2 \mu \mathrm{g} \cdot \mathrm{ml}^{-1}$ using covalent surface chemistries.

In 2007, Leung et al. [64] reported tapered fiber optic biosensors exhibiting strong transmission changes at $1550 \mathrm{~nm}$ when the antibody was bound to the surface and when the antigen bovine serum albumin (BSA) was bound to the antibody. The limit of detection of BSA was found to be $100 \mathrm{fg} / \mathrm{ml}$. A sensing ability at $10 \mathrm{fg} / \mathrm{ml}$ was observed. The response of BTOF was found to be about $1 \mathrm{~h}$ for the antibody immobilization and about $30 \mathrm{~min}$ for the antigen binding.

In 2008, Corres et al. [65] reported a new biosensor which has been developed by coating a tapered optical fiber with an anti-gliadin antibodies (AGAs) sensitive nanofilm using the electrostatic self-assembled (ESA) monolayer technique in order to aid the diagnosis of celiac disease. The minimum detection limit of the sensor was $1 \mathrm{ppm}$.

In the same year, Leung et al. [41] reported the detection of a model protein BSA using antibody-immobilized tapered fiber optic biosensors at $1310 \mathrm{~nm}$ and $1550 \mathrm{~nm}$ under stagnant and flow conditions. In this study, the detection of the BSA in a flow cell configuration was examined because flow reduced non-specific adsorption of contaminating proteins, eliminated transmission changes due to mechanical movements, and allowed for quick switching between samples. Detection experiments were conducted by immobilizing antibody to the BSA on the tapered fiber surface, then exposing them to $1 \mathrm{pg} / \mathrm{mL}-10 \mathrm{ng} / \mathrm{mL}$ of the BSA at $0.5 \mathrm{ml} / \mathrm{min}$ and measuring the transmission at $1310 \mathrm{~nm}$ and $1550 \mathrm{~nm}$.
In 2010, Zibaii et al. [66] reported the real-time monitoring attachment of model protein bovine serum albumin to the antibody-immobilized surface of the NATOF biosensor. The surfaces of the tapers were modified, as shown in Fig. 15, with an amine group to allow for the formation of a covalent bond between the amine and one of the carboxylic groups of the antibody. The attachment of the BSA to the antibody-immobilized surface of the taper was monitored by transmission of a 1558.17-nm DFB laser through the tapered fiber. Figure 16 shows the experimental setup. The NATOF sensor was housed in a specially constructed holder in which exposure of liquid materials like Anti-BSA and BSA occurred in different parts of the experiment. Figure 17(a) shows the typical transmission response of the sensor for reaction of the anti-BSA with the amine-modified taper surface. It was seen that the transmission gradually increased and reached saturated levels. Saturation usually occurred within $2 \mathrm{~h}$. During the immobilization, bonding of the antibody to the fiber surface resulted in a small increase in the fiber diameter as well as the change in the RI on the taper surface.

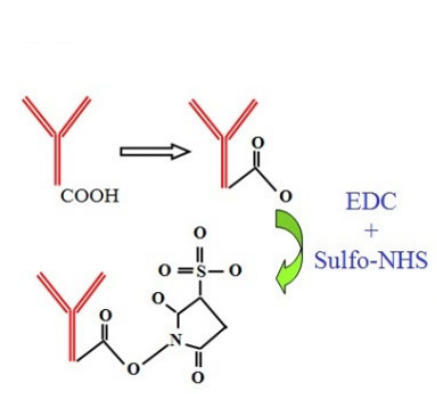

(a)

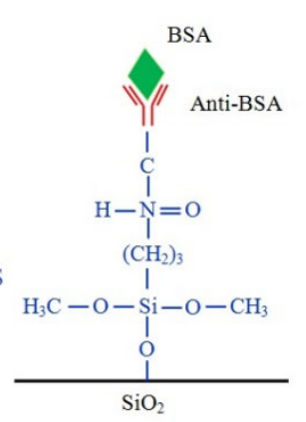

(b)
Fig. 15 Chemical structure of (a) the activated anti-BSA with EDC and Slfu-NHS and (b) immobilization of the anti-BSA on the taper fiber.

This type of surface reaction was analogous to adsorption of a protein onto a surface and could be modeled with the Langmuir model of adsorption. The size of the antibody was about $13 \mathrm{~nm} \times 6 \mathrm{~nm}$, thus the taper diameter was likely to change non-uniformly by $12 \mathrm{~nm}$ to $26 \mathrm{~nm}$, and such a change 
was expected to alter the transmission characteristics.

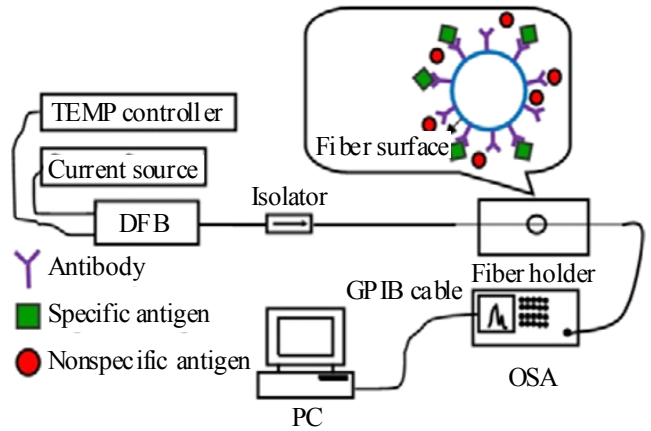

Fig. 16 Experimental setup proposed for BSA-anti BSA interaction [65].

Both the attachment and release responses are shown in Figs. 17(b) and 17(d) for three different concentrations of the BSA in $100 \mathrm{ng} / \mathrm{ml}, 100 \mathrm{pg} / \mathrm{ml}$, and $100 \mathrm{fg} / \mathrm{ml}$. When the BSA was injected into the

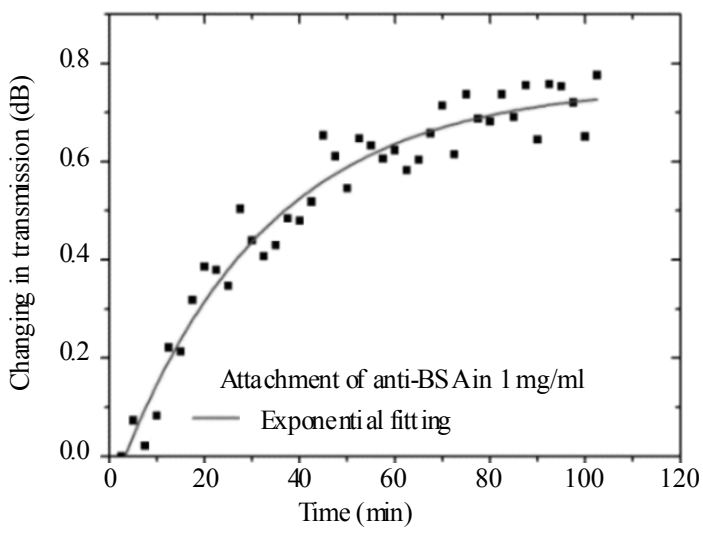

(a)

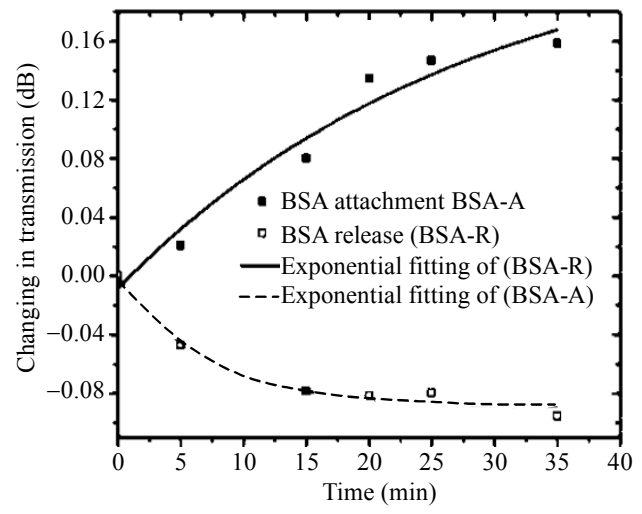

(c) sample holder, transmission decreased due to a change in the surface RI caused by the presence of the BSA. A low pH PBS-A was then added to change the conformation of the proteins so as to loosen the binding between the antibody to the BSA and the BSA. When the BSA was loosened and released from the antibody, transmission increased back almost to the starting value. Similar to the antibody attachment to the fiber surface, the binding of the BSA and anti-BSA appeared to follow the Langmuir adsorption model. Based on the obtained data, the rate constant for anti-BSA adsorption on the tapered surface was found to be $0.03 \mathrm{~min}^{-1}$. An estimate of the antibody/antigen surface coverage of the fiber could be made with a few simplifying assumptions.

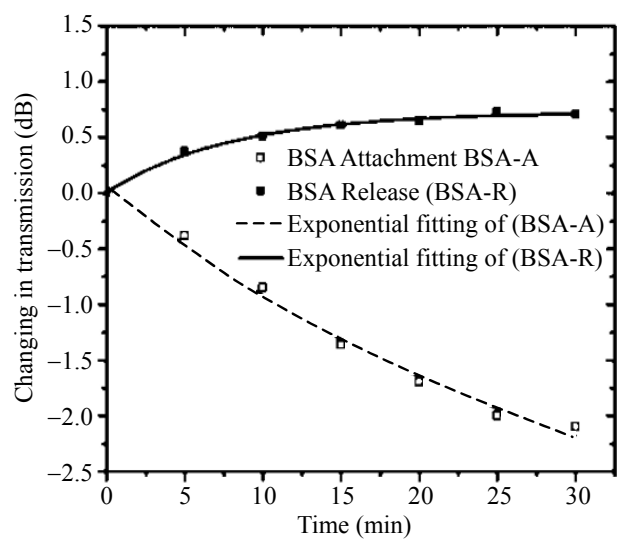

(b)

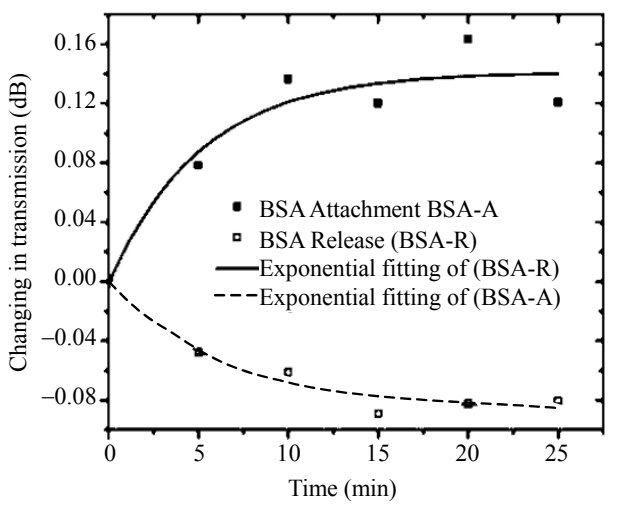

(d)

Fig. 17 Attachment and release responses of the NATOF biosensor: (a) transmission change in dB vs. time for antibody immobilization, (b) BSA attachment and release of $100 \mathrm{ng} / \mathrm{ml}$, (c) $100 \mathrm{pg} / \mathrm{ml}$ sample, and (d) $100 \mathrm{fg} / \mathrm{ml} \mathrm{sample}$. 
Using a convergent length of $400 \mu \mathrm{m}$, the waist length of $100 \mu \mathrm{m}$, divergent length of $1000 \mu \mathrm{m}$, and waist diameter of $12 \mu \mathrm{m}$ were estimated that it would require $6.8 \times 10^{9}$ molecules to completely cover the taper surface if the uniform coverage was assumed. A $200-\mu \mathrm{L}$ sample containing $10 \mathrm{pg} / \mathrm{ml}$ of the BSA had enough BSA molecules to cover less than $0.03 \%$ of the taper surface. In the ideal case, it would require about $3.8 \mathrm{ng} / \mathrm{ml}$ of the $200-\mu 1$ BSA sample to saturate the surface of the fiber. Due to the likelihood that the antibody coverage of the surface was likely to be less than $100 \%$, it was suggested that the concentration required for saturation was less than $4 \mathrm{ng} / \mathrm{ml}$.

\subsubsection{DNA detection}

In 2008, Leung et al. [67] reported detecting DNA hybridization using the intensity-based tapered fiber biosensor in near-IR wavelengths. The taper regions were coated with $50 \mathrm{~nm}$ of gold. The tapered fiber surface was immobilized with 15-mer ssDNA with a C6 extension and a thiol group. Then, the complementary 10-mer ssDNA samples were allowed to flow in from low concentration to high concentration ( $750 \mathrm{fM}$ to $7.5 \mathrm{nM})$, and the resulting transmission changes were recorded. It was shown that $750 \mathrm{fM}$ of complementary DNA could be detected. This sensor was able to distinguish between complementary DNA from DNA with a single nucleotide mismatch in the middle position. In 2010, Zibaii et al. [68] reported a NATOF biosensor-based RI measurement for studying of bimolecular interactions including the ssDNA-ssDNA interaction. The NATOF sensor was immobilized with 25-mer DNA with the PLL solution. The hybridization response of complementary strands was measured at three concentrations of $200 \mathrm{nM}, 500 \mathrm{nM}, 1000 \mathrm{nM}$ of the ssDNA target solution. Figure 18 shows the optical response of the NATOF sensor for probe immobilization and DNA hybridization. The red-shifts and Longmuir behavior of the signal with time were due to an increase in the RI of the external media by hybridizing target ssDNA. As shown in Fig. 19 by plotting equilibrium response $\left(R_{\text {eq }}\right)$ per target concentration $(C)$ versus equilibrium response, a straight line was obtained, whose binding constant could be calculated. In this experiment, the binding constant for ssDNA-ssDNA interaction was measured to be $4.632 \times 10^{6} \mathrm{M}^{-1}$.

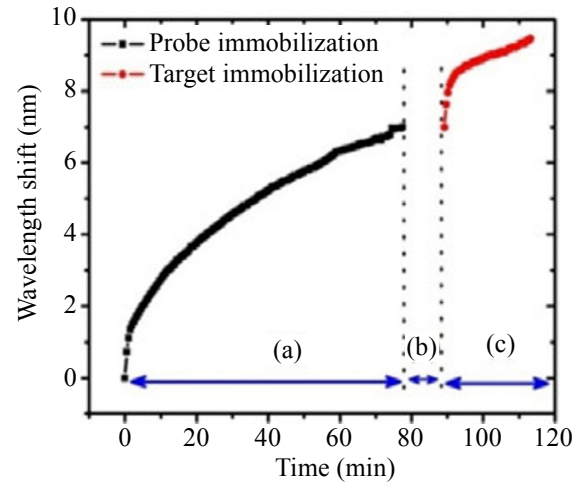

Fig. 18 Wavelength shifts with time for (a) probe immobilization, (b) washing process, and (c) DAN hybridization.

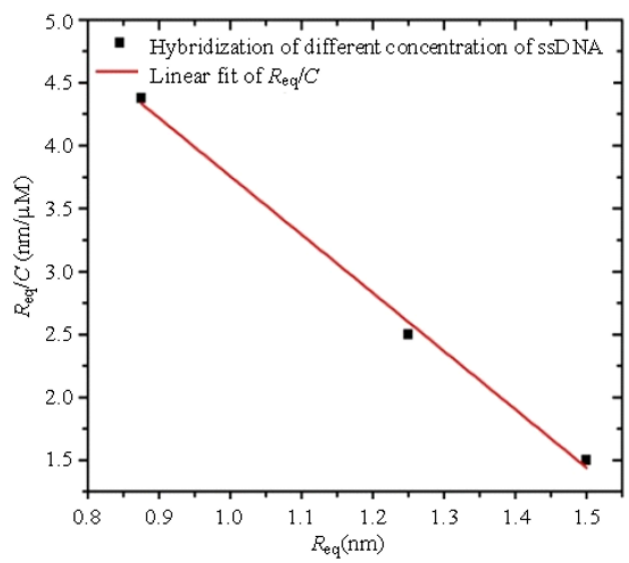

Fig. 19 Plot of $R_{\text {eq }} / C$ versus $R_{\text {eq }}[68]$.

\section{Future remarks}

BTOFs can serve as basic elements for optical sensing or for light input/output in miniature photonic sensors. Due to their favorable properties of high fractional EWFs, low loss, and high flexibility for optical sensing, NATOF sensors may offer advantages of high sensitivity, fast response, small footprint, high spatial resolution, and low detection limits. In addition, the micro scale sizes of these sensors make them possible to integrate the 
NATOF biosensors with microfluidic or nanofluidic chips for practical applications that require very small quantities of samples. The adiabatic tapered fiber is also used for coupling light to other photonic devices. One area of the adiabatic tapered fiber research is the development of high sensitive SPR biosensors which are operated in longer wavelengths. The detection principle is another area of the fiber biosensor technology which was successfully explored: it appears that fluorescent-based sensors have been used to a limited extent in the detection of biological parameters. However, for protein and DNA detection, the use of the fluorescence tapered fiber optic biosensor is necessary to detect low levels of biomolecules at ultraviolet (UV) and visible wavelengths. NATOF biosensor is an attractive method for label free sensing because it does not require any labeling. As a result, there has been a shift in protein and increasingly DNA detection by using the NATOF sensor. After surveying the large number of studies on the tapered fiber optic biosensor over the past 10 years, it appears that detection of the pathogens, drug screening based antibodies, and DNA will continue to flourish along the advancement of medical diagnostics, clinical applications, a safe environment and food supply. As shown in this review, because of their miniature sensing probe, label-free direct detection and high sensitivity, NATOF biosensors will remain a popular choice among researchers and practitioners for detection of biological agents.

Open Access This article is distributed under the terms of the Creative Commons Attribution License which permits any use, distribution, and reproduction in any medium, provided the original author(s) and source are credited.

\section{References}

[1] M. D. Marazuela and M. C. Moreno-Bondi,
"Fiber-optic biosensors - an overview," Analytical and Bioanalytical Chemistry, vol. 372, no. 5-6, pp. 664-682, 2002.

[2] A. M. Valadez, C. A. Lana, S. I. Tu, M. T. Morgan, and A. K. Bhunia, "Evanescent wave fiber optic biosensor for Salmonella detection in food," Sensors, vol. 9, no. 7, pp. 5810-5824, 2009.

[3] A. W. Snyder and J. D. Love, Optical Waveguide Theory. London: Chapman and Hall, 1983.

[4] W. J. Stewart and J. D. Love, "Design limitation on tapers and couplers in singlemode fiber tapers," in Proc. ECOC 85, Venice, Oct. 1-4, pp. 559-562,1985.

[5] J. D. Love and W. M. Henry, "Quantifying loss minimisation in single-mode fiber tapers," Electronics Letters, vol. 22, no. 17, pp. 912-914, 1986.

[6] M. Sumetsky, Y. Dulashko, and A. Hale, "Fabrication and study of bent and coiled free silica nanowires: self-coupling microloop optical interferometer," Optics Express, vol. 12, no. 15, pp. 3521-3531, 2004.

[7] L. Shi, X. Chen, H. Liu, Y. Chen, Z. Ye, W. Liao, et al., "Fabrication of submicron-diameter silica fibers using electric strip heater," Optics Express, vol.14, no. 12, pp. 5055-5060, 2006.

[8] E. J. Zhang, W. D. Sacher, and J. K. Poon, "Hydrofluoric acid flow etching of low-loss subwavelength-diameter biconical fiber tapers," Optics Express, vol. 18, no. 21, pp. 22593-22598, 2010.

[9] S. Pricking and H. Giessen, "Tapering fibers with complex shape," Optics Express, vol. 18, no. 4, pp. 3426-3437, 2010.

[10] V. P. Minkovich and D. Monzón-Hernández, "Microstructured optical fiber coated with thin films for gas and chemical sensing," Optics Express, vol. 14, no. 18, pp. 8413-8418, 2006.

[11] J. Ju, L. Ma, and W. Jin, "Photonic bandgap fiber tapers and interferometric sensors," in Proc. SPIE, vol. 7503, pp.75035B-1-75035B-4, 2009.

[12] M. I. Zibaii, H. Latifi, K. Karami,M. Gholami, S. M. Hosseini, and M. H. Ghezelayagh, "Non-adiabatic tapered optical fiber sensor for measuring the interaction between $\alpha$-amino acids in aqueous carbohydrate solution," Measurement and Science Technology, vol. 21, no. 10, pp. 105801, 2010.

[13] J. D. Love, W. M. Henry, W. J. Stewart, R. J. Black, S. Lacroix, and F. Gonthier, "Tapered single-mode fibers and devices: part 1. adiabaticity criteria," IEE Proc. J. Optoelectronics, vol. 138, no. 5, pp. 343-354, 1991.

[14] R. J. Black, S. Lacroix, F. Gonthier, and J. D. Love, "1991 tapered single mode fibers and devices: part 2. 
experimental and theoretical quantification," IEE Proc. J Optoelectronics, vol. 138, no. 5, pp. 355-364, 1991.

[15] I. M. White, H. Oveys, and X. Fan, "Liquid-coreoptical ring-resonator sensors," Optics Letters, vol.31, no. 9, pp. 1319-1321, 2006.

[16] A. M. Armani and K. J. Vahala, "Heavy water detection using ultra-high- $Q$ microcavities," Optics Letters, vol. 31, no. 12, pp. 1896-1898, 2006.

[17] D. Keng, S. R. McAnanama, I. Teraoka, and S. Arnold, "Resonance fluctuations of a whispering gallery mode biosensor by particles undergoing Brownian motion," Applied Physics Letters, vol. 91, no. 10, pp. 103902-1-103902-3, 2007.

[18] J. Yi, C. Y. Jao, I. L. N. Kandas, B. Liu, Y. Xu, and H. D. Robinson, "Irreversible adsorption of gold nanospheres on fiber optical tapers and microspheres," Applied Physics Letters, vol. 100, no. 15, pp.153107-1-153107-4, 2012.

[19] F. Vollmer, D. Braun, A. Libchaber, M. Khoshsima, I. Teraoka, and S. Arnold, "Protein detection by optical shift of a resonant microcavity," Applied Physics Letters, vol. 80, no. 21, pp. 4057-4059, 2002.

[20] K. Q. Kieu and M. Mansuripur, "Biconical fiber taper sensor," IEEE Photonics Technology Letters, vol. 18, no. 21, pp. 2239-2241, 2006.

[21] P. Datta, C. Matias, C. Aramburu, A. Bakas, M. Lopez-Amo, and J. M. Oton, "Tapered optical fiber temperature sensor," Microwave Optical Technology Letters, vol. 11, no. 2, pp. 93-95, 1996.

[22] J. M. Corres, J. Bravo, I. R. Matias, and F. J. Arregui, "Nonadiabatic tapered single-mode fiber coated with humidity sensitive nanofilms," IEEE Photonics Technology Letters, vol. 18, no. 8, pp.935-937, 2006.

[23] B. S. Kawasaki, K. O. Hill, and R. C. Lamont, "Biconical taper single-mode fiber coupler," Optics Letter, vol. 6, no. 7, pp. 327-328, 1981.

[24] W. Bums, M. Abebe, C. Villarruel, and R. Moeller, "Loss mechanisms in single-mode tapers," Journal of Lightwave Technology, vol. 4, no. 6, pp. 608-613, 1986.

[25] L. C. Bobb, P. M. Shankar, and H. D. Krumboltz., "Bending effects in biconically tapered single-mode fibers," Journal of Lightwave Technology, vol. 8, no. 7, pp. 1084-1090, 1990.

[26] F. Gonthier, A. Henault, S. Lacroix, R. J. Black, and J. Bures, "Mode coupling in nonuniform fibers: comparison between coupled-mode theory and finite-difference beam-propagation method simulations," Optical Society of America B: Optical
Physics, vol. 8, no. 2, pp. 416-421, 1991.

[27] P. N. Moar, S. T. Huntington, J. Katsifolis, L. W. Cahill, A. Roberts, and K. A. Nugent, "Fabrication, modeling, and direct evanescent field measurement of tapered optical fiber sensors," Journal of Applied Physics, vol. 85, no. 7, pp. 3395-3398, 1999.

[28] A. J. Fielding and C. C. Davis, "Tapered single-mode optical fiber evanescent coupling," IEEE Photonics Technology Letters, vol. 14, no. 1, pp. 53-55, 2002.

[29] I. R. Matias, C. F. Valdivielso, F. J. Arregui, C. Bariain, and M. L. Amo, "Transmitted optical power through a tapered single-mode fiber under dynamic bending effects," Fiber and Integrated Optics, vo. 22, no. 3, pp. 173-187, 2003.

[30] M. Ahmad and L. L. Hench, "Effect of taper geometries and launch angle on evanescent wave penetration depth in optical fibers," Biosensors and Bioelectronics, vol. 20, no. 7, pp. 1312-1319, 2005.

[31] A. J. C. Tubb, F. P. Payne, R. Millington, and C. R. Lowe, "Singlemode optical fiber surface plasma wave chemical sensor," Electronics Letters, vol. 31 no. 20, pp. 1770-1771,1995.

[32] Ó. Esteban, N. Díaz-Herrera, M. C. Navarrete, and A. González-Cano, "Surface plasmon resonance sensors based on uniform-waist tapered fibers in a reflective configuration," Applied Optics, vol. 45, no. 28, pp. 7294-7298, 2006.

[33] R. K. Verma, A. K. Sharma, and B. D. Gupta, "Surface plasmon resonance based tapered fiber optic sensor with different taper profiles," Optics Communications, vol. 281, no. 6, pp. 1486-1491, 2008.

[34] N. Díaz-Herrera, A. González-Cano, D. Viegas, J. Luís. Santos, and M. C. Navarrete, "Refractive index sensing of aqueous media based on plasmonic resonance in tapered optical fibers operating in the $1.5 \mu \mathrm{m}$ region," Sensors and Actuators B: Chemical, vol. 146, no. 1, pp. 195-198.

[35] N. Díaz-Herrera, O. Esteban, M. C. Navarrete, A. González-Cano, E. Benito-Pena, and G. Orellana, "Improved performance of SPR sensors by a chemical etching of tapered optical fibers," Optics and Lasers in Engineering, vol. 49, no. 8, pp. 1065-1068, 2011.

[36] A. Kumar, T. V. B. Subrahmonium, A. D. Sharma, K. Thyagarajan, B. P. Pal, and I. C. Goyal, "Novel refractometer using a tapered optical fiber," Electronics Letters, vol. 20, no. 13, pp. 534-535, 1984.

[37] J. Villiatoro, D. Monzoon-Hernandez, and D. Talavera, 
"High resolution refractive index sensing with cladded multimode tapered optical fiber," Electronics Letters, vol. 40, no. 2, pp.106-107, 2004.

[38] P. Wang, G. Brambilla, M. Ding, Y. Semenova, and Q. Wu, G. Farrell, "High-sensitivity, evanescent field refractometric sensor based on a tapered, multimode fiber interference," Optics Letters, vol. 36, no. 12, pp. 2233-2235, 2011.

[39] P. Polynkin, A. Polynkin, N. Peyghambarian, and M. Mansuripur, "Evanescent field-based optical fiber sensing device for measuring the refractive index of liquids in microfluidic channels," Optics Letters, vol. 30, no. 11, pp. 1273-1275, 2005.

[40] J. Arrue, F. Jiménez, G. Aldabaldetreku, G. Durana, J. Zubia, M. Lomer, et al., "Analysis of the use of tapered graded-index polymer optical fibers for refractive-index sensors," Optics Express, vol. 16, no. 21, pp. 16616-16631, 2008.

[41] A. Leung, P. Mohana Shankar, and R. Mutharasan, "Model protein detection using antibody-immobilized tapered fiber optic biosensors (TFOBS) in a flow cell at $1310 \mathrm{~nm}$ and $1550 \mathrm{~nm}$," Sensors and Actuators B: Chemical, vol. 129, no. 2, pp. 716-725, 2008.

[42] G. Cohoon, C. Boyter, M. Errico, K. Vandervoort, and E. Salik, "Enhancing sensitivity of biconical tapered fiber sensors with multiple passes through the taper," Optical Engineering, vol. 49, no. 3, pp. 034401-5, 2010.

[43] H. A. Rahman, S. W. Harun, M. Yasin, S. W. Phang, S. S. A. Damanhuri, H. Arof, et al., "Tapered plastic multimode fiber sensor for salinity detection," Sensors and Actuators A: Physical, vol. 171, no. 2, pp. 219-222, 2011.

[44] C. Beres, F. V. B. Nazaré, N. C. C. Souza, M. A. L. Miguel, and M. M. Werneck, "Tapered plastic optical fiber-based biosensor - tests and application," Biosensors and Bioelectronics, vol. 30, no. 1, pp. 328-332, 2011.

[45] J. F. Ding, A. P. Zhang, L. Y. Shao, J. H. Yan, and S. He, "Fiber-taper seeded long-period grating pair as a highly sensitive refractive-index sensor," IEEE Photonics Technology Letters, vol. 17, no. 6, pp. 1247-1249, 2005.

[46] T. Allsop, F. Floreani, K. P. Jedrzejewski, P. V. S. Marques, R. Romero, D. J. Webb, et al., "Spectral characteristics of tapered LPG device as a sensing element for refractive index and temperature," Journal of Lightwave Technology, vol. 24, no. 2, pp. 870-878, 2006.

[47] D. Grobnic, S. J. Mihailov, D. Huimin, and C. W. Smelser, "Bragg grating evanescent field sensor made in biconical tapered fiber with femtosecond IR radiation," IEEE Photonics Technology Letters, vol. 18, no. 1, pp. 160-162, 2006.

[48] W. Liang, Y. Y. Huang, Y. Xu, K. L. Reginald, and Y. Amnon, "Highly sensitive fiber Bragg grating refractive index sensors," Applied Physics Letters, vol. 86, no. 15, pp. 151122-1-151122-3, 2005.

[49] X. Fang, C. R. Liao, and D. N. Wang "Femtosecond laser fabricated fiber Bragg grating in microfiber for refractive index sensing," Optics Letters, vol. 35, no. 7, pp. 1007-1009, 2010.

[50] M. I. Zibaii, O. Frazão, H. Latifi, and P. A. S. Jorge, "Controlling the sensitivity of refractive index measurement using a tapered fiber loop mirror," IEEE Photonics Technology Letters, vol. 23, no. 17, pp. 1219-1221, 2011.

[51] O. Frazão, J. M. Baptista, and J. L. Santos, "Recent advances in high-birefringence fiber loop mirror sensors," Sensors, vol. 7, no. 11, pp. 2970-2983, 2007.

[52] W. E. Moerner, "New directions in single-molecule imaging and analysis," Proceedings of the National Academy of Sciences of the United States of America, vol. 104, no. 31, pp. 12596-12602, 2007.

[53] W. G. Cox and V. L. Singer, "Fluorescent DNA hybridization probe preparation using amine modification and reactive dye coupling," Biotechniques, vol. 36, no. 1, pp.114-122, 2004.

[54] E. A. James, K. Schmeltzer, and F. S. Ligler, "Detection of endotoxin using an evanescent wave fiber-optic biosensor," Applied Biochemistry and Biotechnology, vol. 60, no. 3, pp. 189-202, 1996.

[55] Z. M. Hale, F. P. Payne, R. S. Marks, C. R. Lowe, C. R. Lowe, and M. M. Levine, "The single mode tapered optical fiber loop immunosensor," Biosensors and Bioelectronics, vol. 11, no. 1-2, pp. 137-148, 1996.

[56] U. Narang, G. P. Anderson, F. S. Ligler, and J. Burans, "Fiber optic-based biosensor for ricin," Biosensors and Bioelectronics, vol. 12, no. 9-10, pp. 937-945, 1997.

[57] S. Pilevar, C. C. Davis, and F. Portugal, "Tapered optical fiber sensor using near infrared fluorophores to assay hybridization," Analytical Chemistry, vol. 70, no. 10, pp. 2031-2037, 1998.

[58] H. S. Haddock, P. M. Shankar, and R. Mutharasan, "Evanescent sensing of biomolecules and cells," Sensors Actuators B: Chemical, vol. 88, no. 1, pp. 67-74, 2003.

[59] A. P. Ferreira, M. M. Werneck, and R. M. Ribeiro, "Development of an evanescent-field fiber optic 
sensor for Escherichia coli O157: H7," Biosensors and Bioelectronics, vol. 16, no. 6, pp. 399-408, 2001.

[60] K. Rijal, A. Leung, P. M. Shankar, and R. Mutharasan, "Detection of vathoizen Escherichia coli O157: H7 AT 70 cells $/ \mathrm{mL}$ using antibody-immobilized biconical tapered fiber sensors," Biosensor and Bioelectronics, vol. 21, no. 6, pp. 871-880, 2005.

[61] D. Maraldo, P. M. Shankar, and R. Mutharasan, "Measuring bacterial growth by tapered fiber and changes in evanescent field," Biosensors and Bioelectronics, vol. 21, no. 7, pp. 1339-1344, 2006.

[62] M. I. Zibaii, A. Kazemi, H. Latifi, M. Karimi Azar, S. M. Hosseini, and M. H. Ghezelaiagh, "Measuring bacterial growth by refractive index tapered fiber optic biosensor," Photochemistry and Photobiology B: Biology, vol. 101, no. 3, pp. 313-320, 2010.

[63] H. Tazawa, T. Kanie, and M. Katayama, "Fiber-optic coupler based refractive index sensor and its application to biosensing," Applied Physics Letters, vol. 91, no. 11, pp. 113901-1-113901-3, 2007.

[64] A. Leung, P. M. Shankar, and R. Mutharasan, "Real-time monitoring of bovine serum albumin at femtogram $/ \mathrm{mL}$ levels on antibody immobilized tapered fibers," Sensors Actuators B: Chemical, vol. 123, no. 2, pp. 888-895, 2007.

[65] J. M. Corres, I. R. Matias, J. Bravo, and F. J. Arregui, "Tapered optical fiber biosensor for the detection of anti-gliadin antibodies," Sensors and Actuators B: Chemical, vol. 135, no. 1, pp. 166-171, 2008.

[66] M. I. Zibaii, H. Latifi, M. Arabsorkhi, A. Kazemi, M. Gholami, M. Karimi Azar, et al., "Biconical tapered optical fiber biosensor for real-time monitoring of bovine serum albumin at femtogram $/ \mathrm{mL}$ levels on antibodyimmobilized tapered fibers," in Proc. SPIE, vol. 7653, pp. 765322, 2010.

[67] A. Leung, P. M. Shankar, and R. Mutharasan, "Label-free detection of DNA hybridization using gold-coated tapered fiber optic biosensors (TFOBS) in a flow cell at $1310 \mathrm{~nm}$ and $1550 \mathrm{~nm}$," Sensors and Actuators B: Chemical, vol. 131, no. 2, pp. 640-645, 2008.

[68] M. I. Zibaii, Z. Taghipour, Z. Saeedian, H. Latifi, M. Gholami, and S. M. Hosseini, "Kinetic study for the hybridization of 25-mer DNA by nonadiabatic tapered optical fiber sensor," in Proc. SPIE-OSA-IEEE, China, Nov. 13-16, vol. 8311, pp. 831109-1-831109-6, 2011. 